Supporting Information

\title{
Biofilm Removal by Reversible Shape Recovery of the Substrate
}

Sang Won Lee ${ }^{1 \perp}$, Joseph Carnicelli', Dariya Getya²,3, Ivan Gitsov²,3, K. Scott Phillips ${ }^{4}$, and Dacheng Ren ${ }^{1,5,6^{*}}$

1Department of Biomedical and Chemical Engineering, Syracuse University, Syracuse, NY 13244, United States

${ }^{2}$ Department of Chemistry, State University of New York - College of Environmental Science and Forestry, Syracuse, NY 13210, United States

${ }^{3}$ The Michael M. Szwarc Polymer Research Institute, Syracuse, NY 13210, United

\section{States}

${ }^{4}$ United States Food and Drug Administration, Office of Medical Products and Tobacco, Center for Devices and Radiological Health, Office of Science and Engineering Laboratories, Division of Biology, Chemistry, and Materials Science, Silver Spring, MD 20993, United States

${ }^{5}$ Department of Civil and Environmental Engineering, Syracuse University, Syracuse, NY 13244, United States

${ }^{6}$ Department of Biology, Syracuse University, Syracuse, NY 13244, United States

${ }^{*}$ Corresponding author:

Dacheng Ren: Phone +1-315-443-4409. Fax+1-315-443-9175. Email: dren@syr.edu 
${ }^{\perp}$ Present address:

Sang Won Lee

United States Food and Drug Administration, Office of Medical Products and Tobacco, Center for

Devices and Radiological Health, Office of Science and Engineering Laboratories, Division of Biology, Chemistry, and Materials Science, Silver Spring, MD 20993 


\section{Contents}

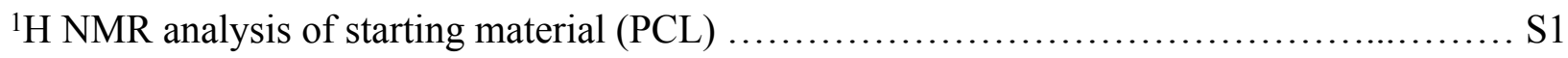

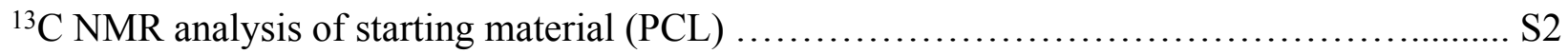

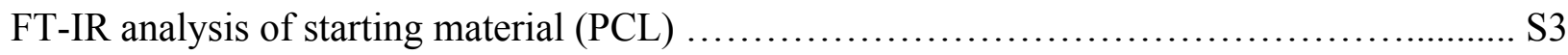

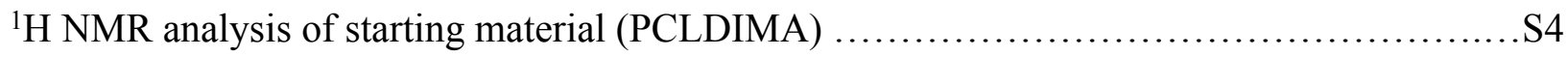

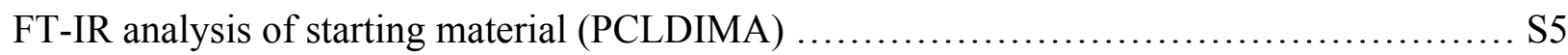

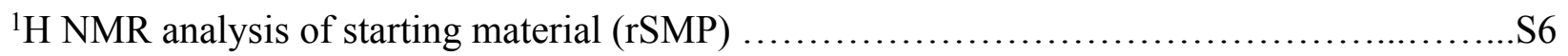

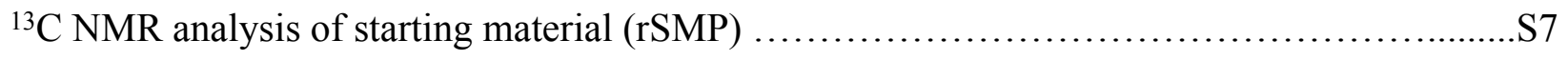

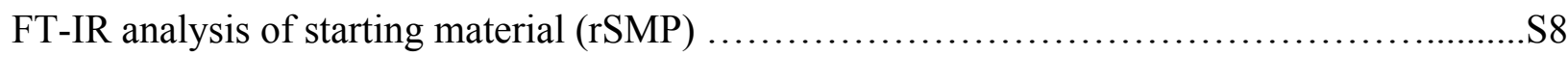

Size-exclusion chromatography (SEC) of PCLDIMA precursors ........................... 9

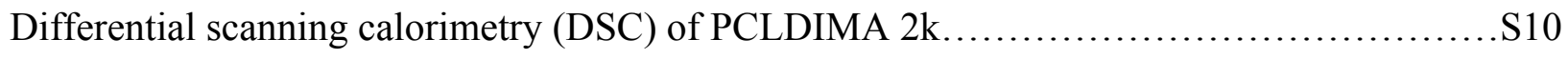

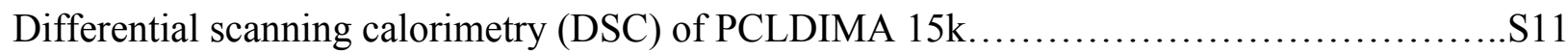

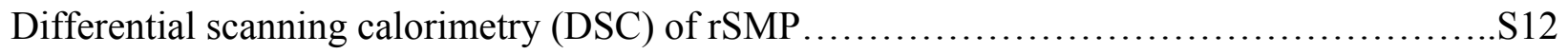




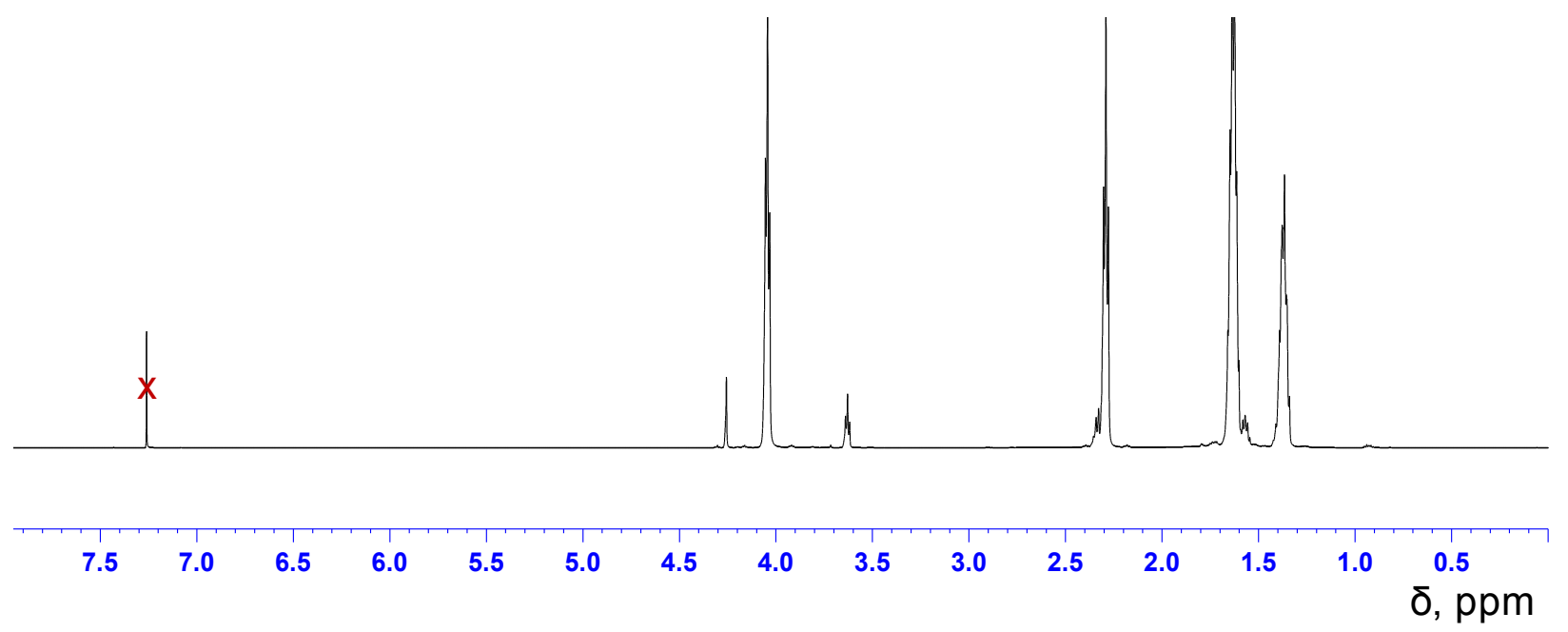

Figure S1. ${ }^{1} \mathrm{H}$ NMR spectrum of PCL $15 \mathrm{k}$ recorded at $600 \mathrm{MHz}$ in $\mathrm{CDCl}_{3}$ at room temperature. $\mathrm{X}$ - solvent signal.

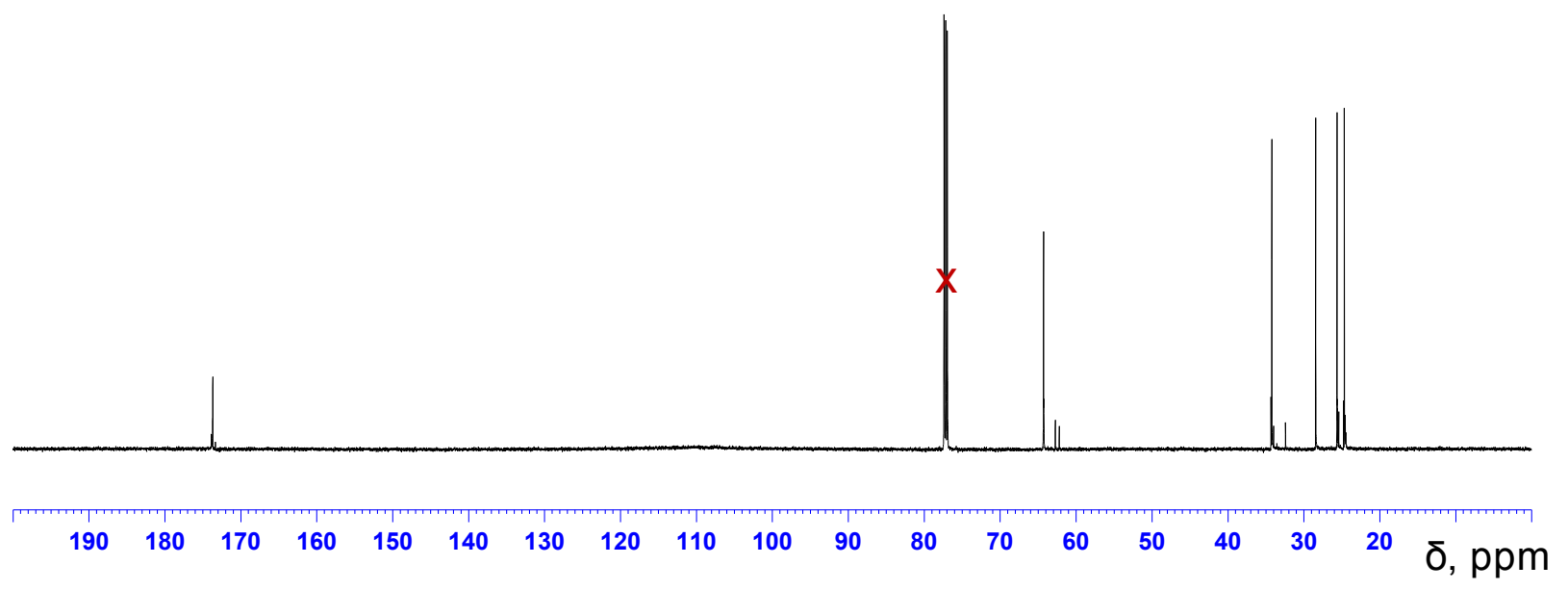

Figure S2. ${ }^{13} \mathrm{C}$ NMR spectrum of PCL $15 \mathrm{k}$ recorded at $152 \mathrm{MHz}$ in $\mathrm{CDCl}_{3}$ at room temperature. $\mathrm{X}$ - solvent signal. 


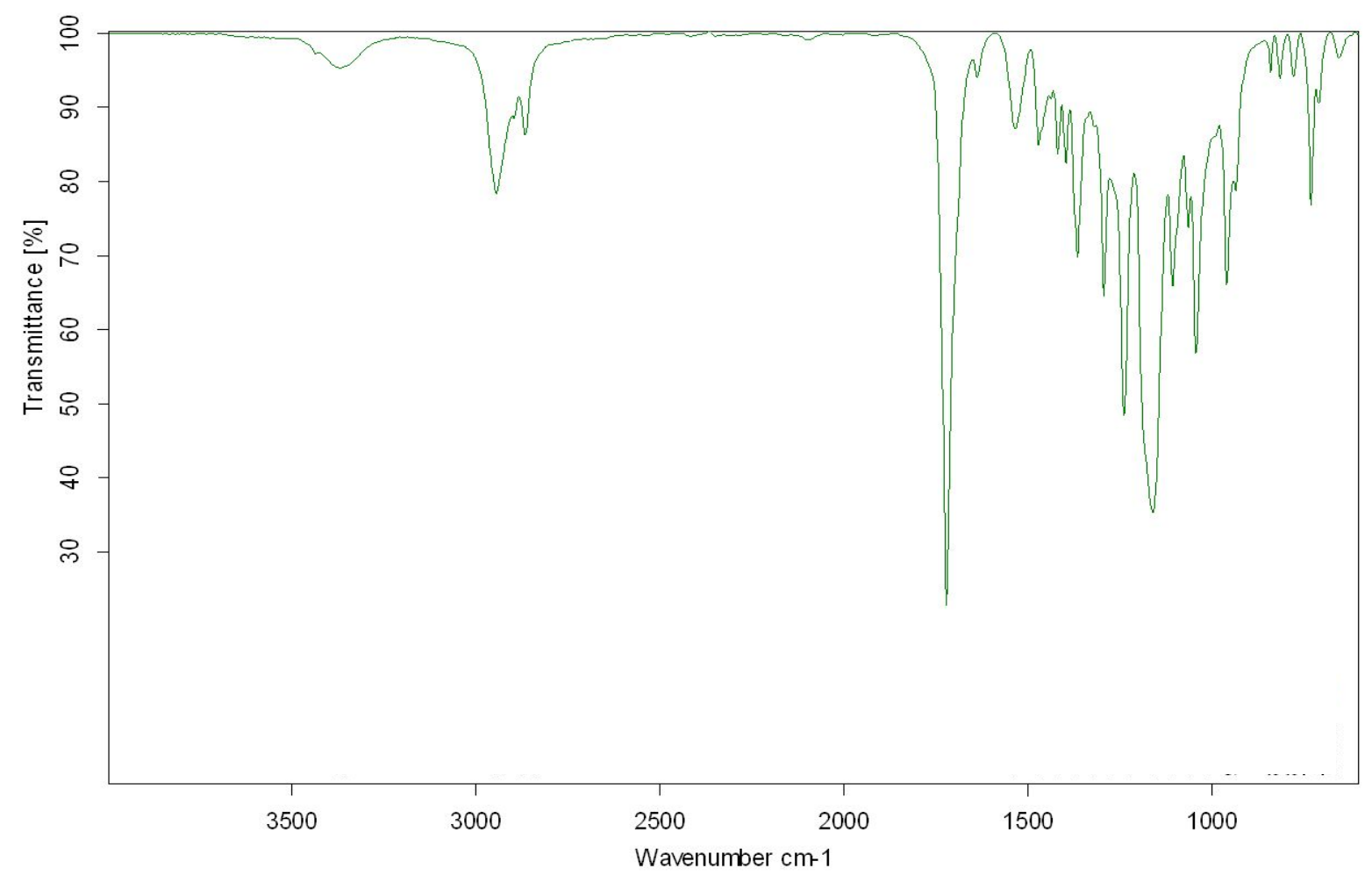

Figure S3. ATR FT-IR spectrum of PCL 15k.

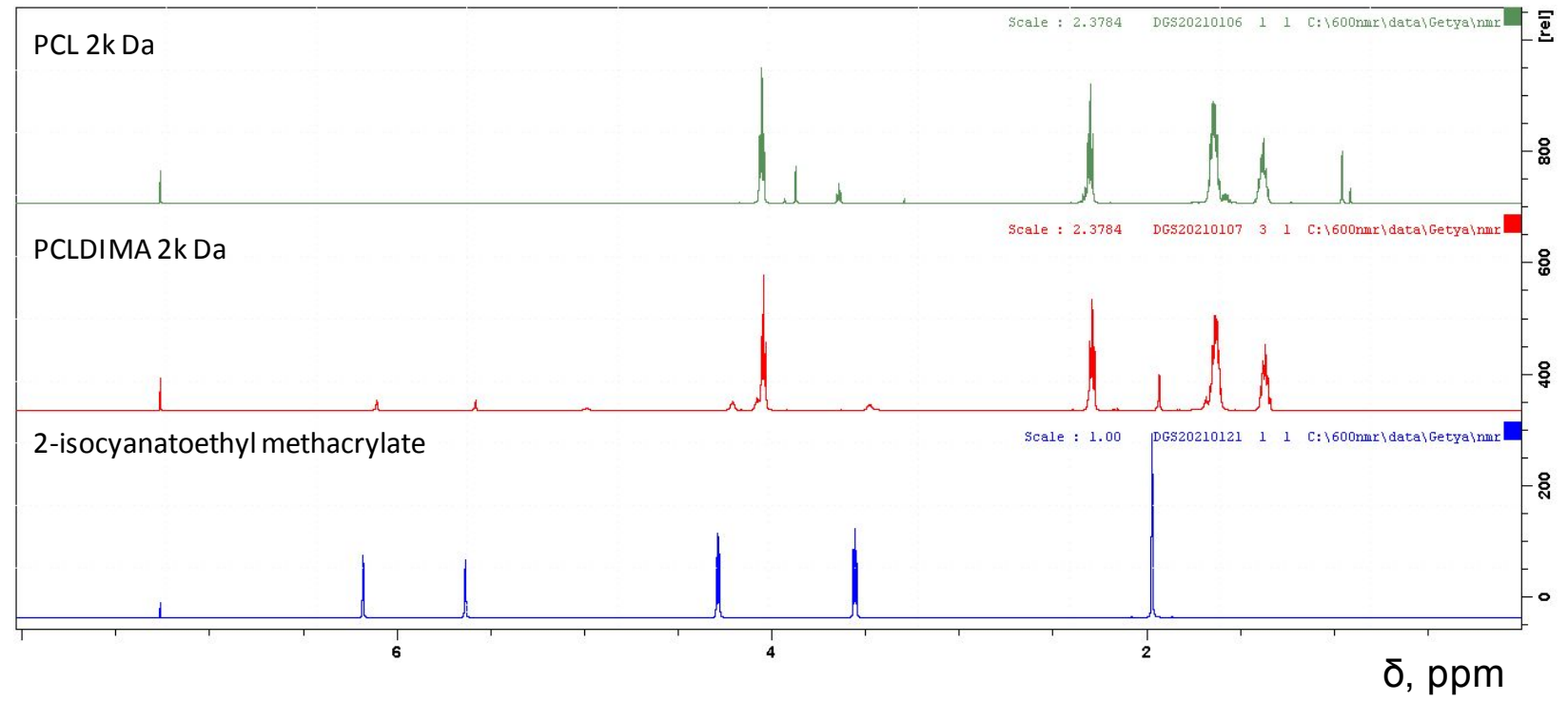

Figure S4. Overlaid $600 \mathrm{MHz}{ }^{1} \mathrm{H}$ NMR spectra of 2-isocyanatoethyl methacrylate (bottom, blue trace), PCLDIMA 2k (middle, red trace), and PCL2k (top, green trace). 


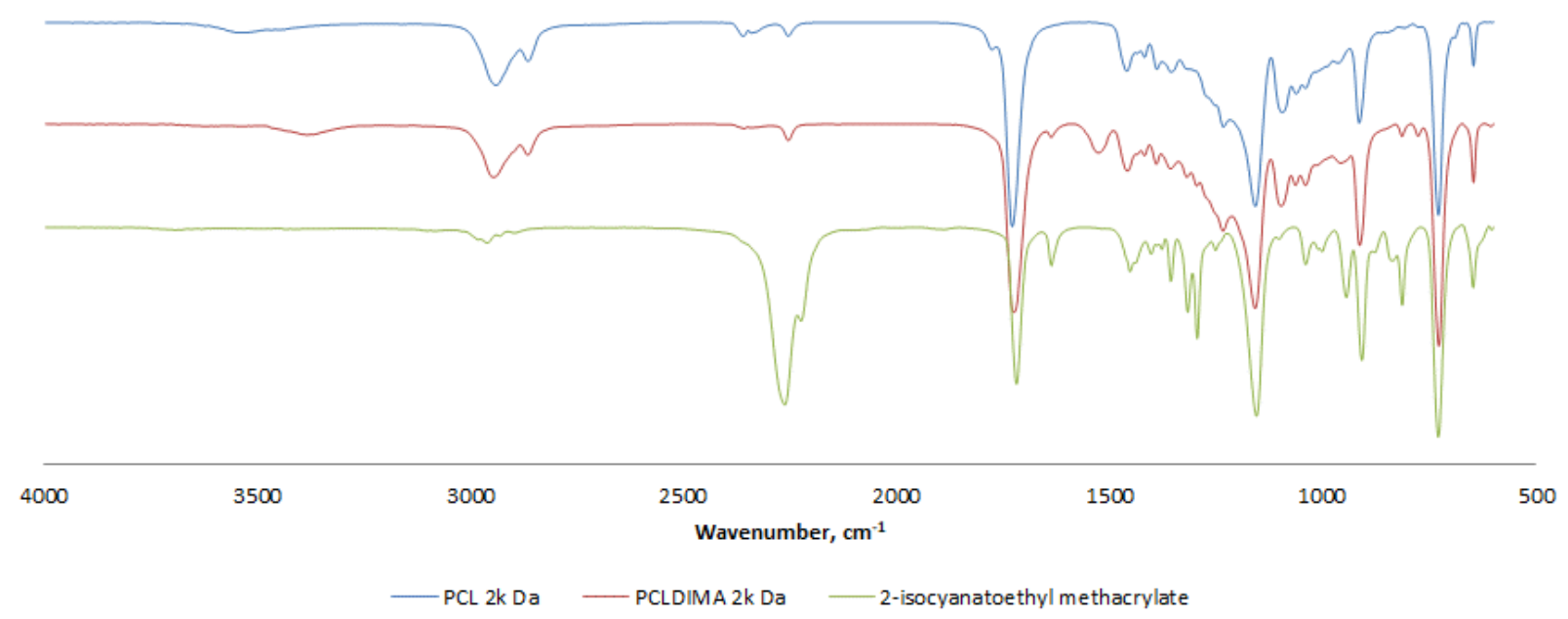

Figure S5. Overlaid ATR FT-IR spectra of 2-isocyanatoethyl methacrylate (bottom, green trace), PCLDIMA 2k (middle, red trace), and PCL2k (top, blue trace).

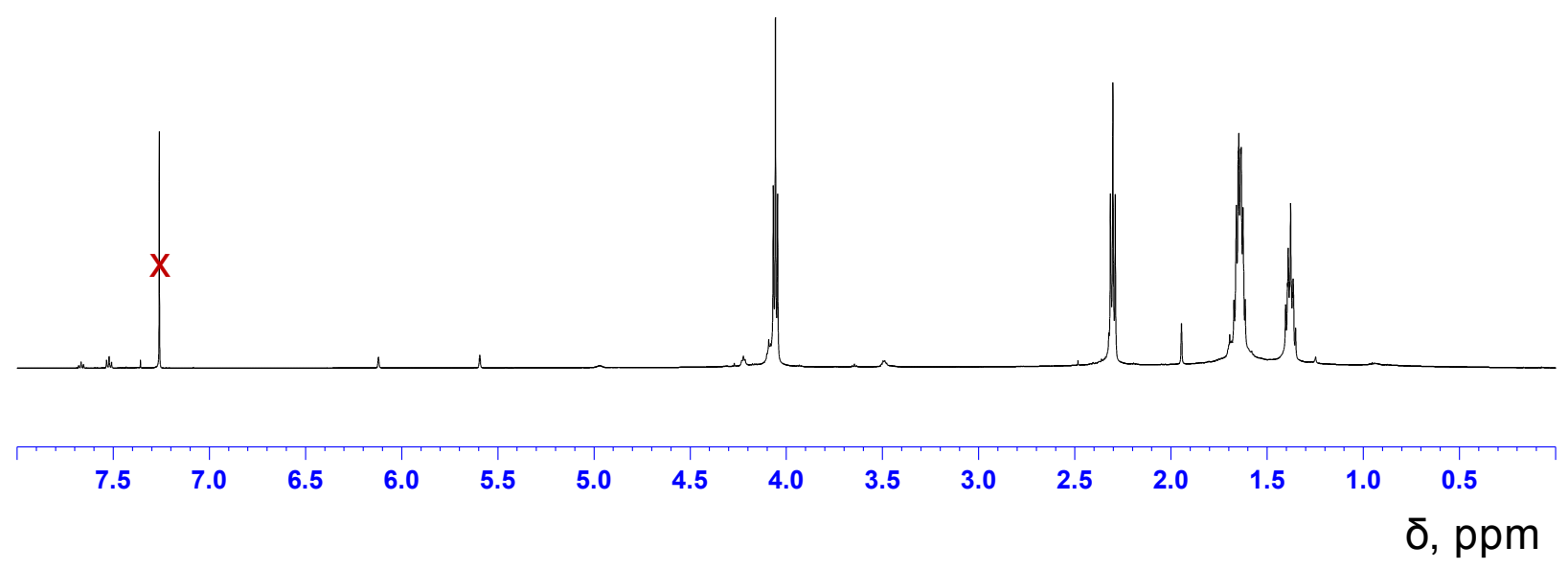

Figure S6. ${ }^{1} \mathrm{H}$ NMR spectrum rSMP recorded at $600 \mathrm{MHz}$ in $\mathrm{CDCl}_{3}$ at room temperature. $\mathrm{X}-$ solvent signal. 


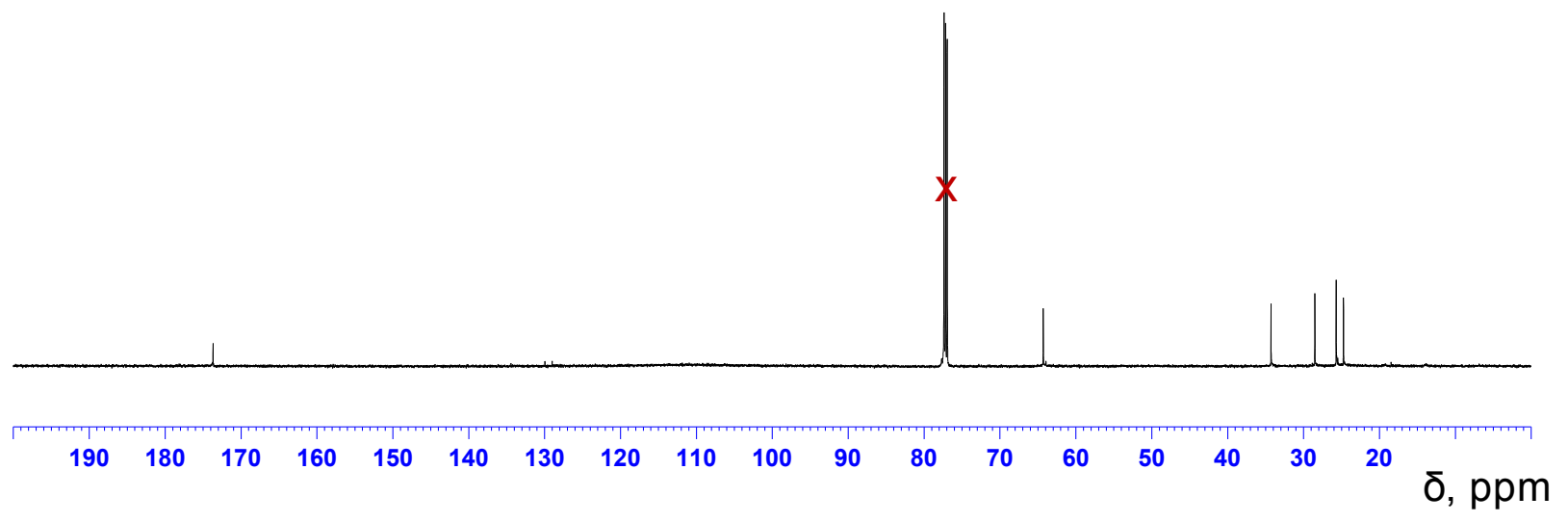

Figure S7. ${ }^{13} \mathrm{C}$ NMR spectrum of rSMP recorded at $152 \mathrm{MHz}$ in $\mathrm{CDCl}_{3}$ at room temperature. $\mathrm{X}-$ solvent signal.

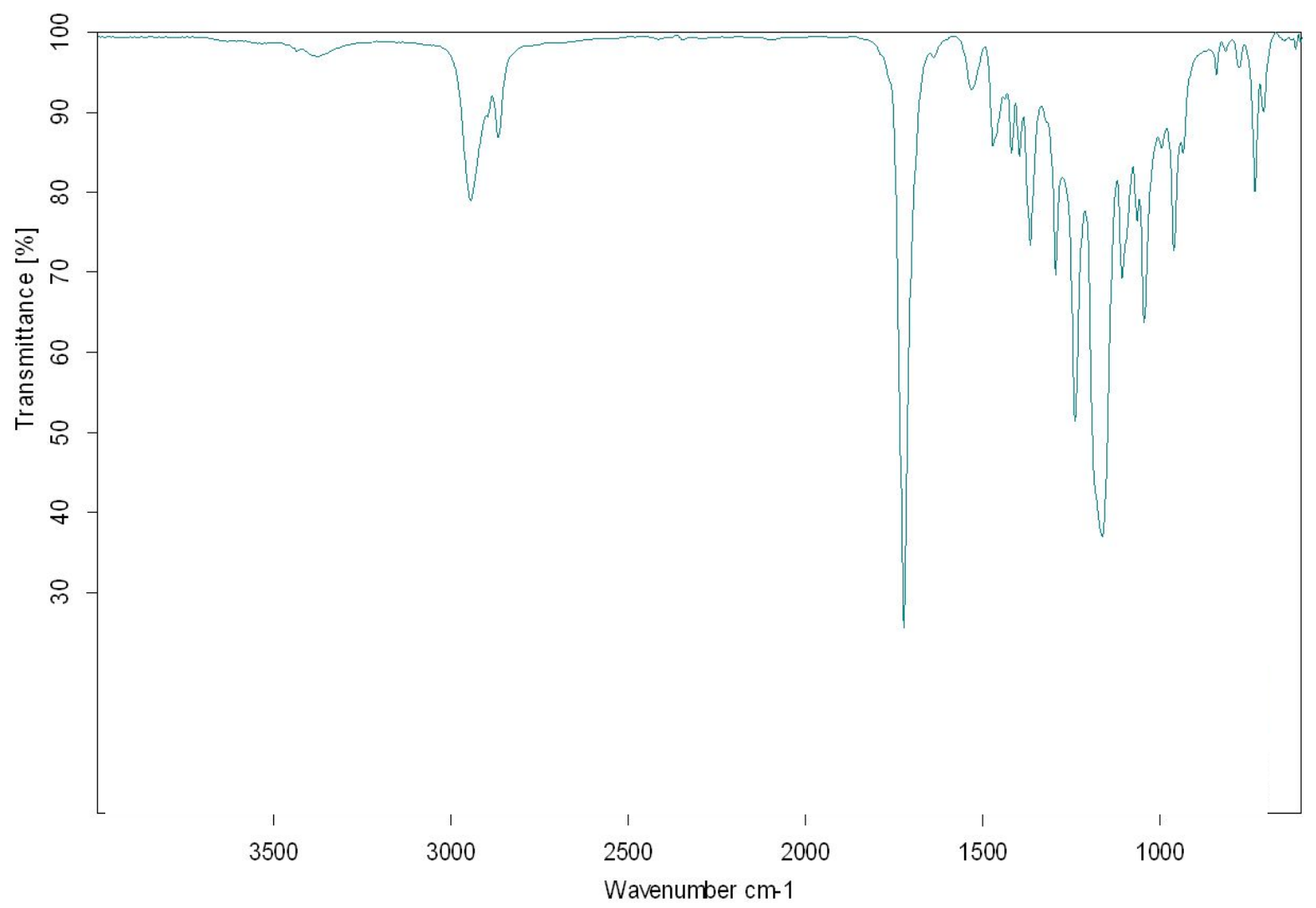

Figure S8. ATR FT-IR spectrum of rSMP. 


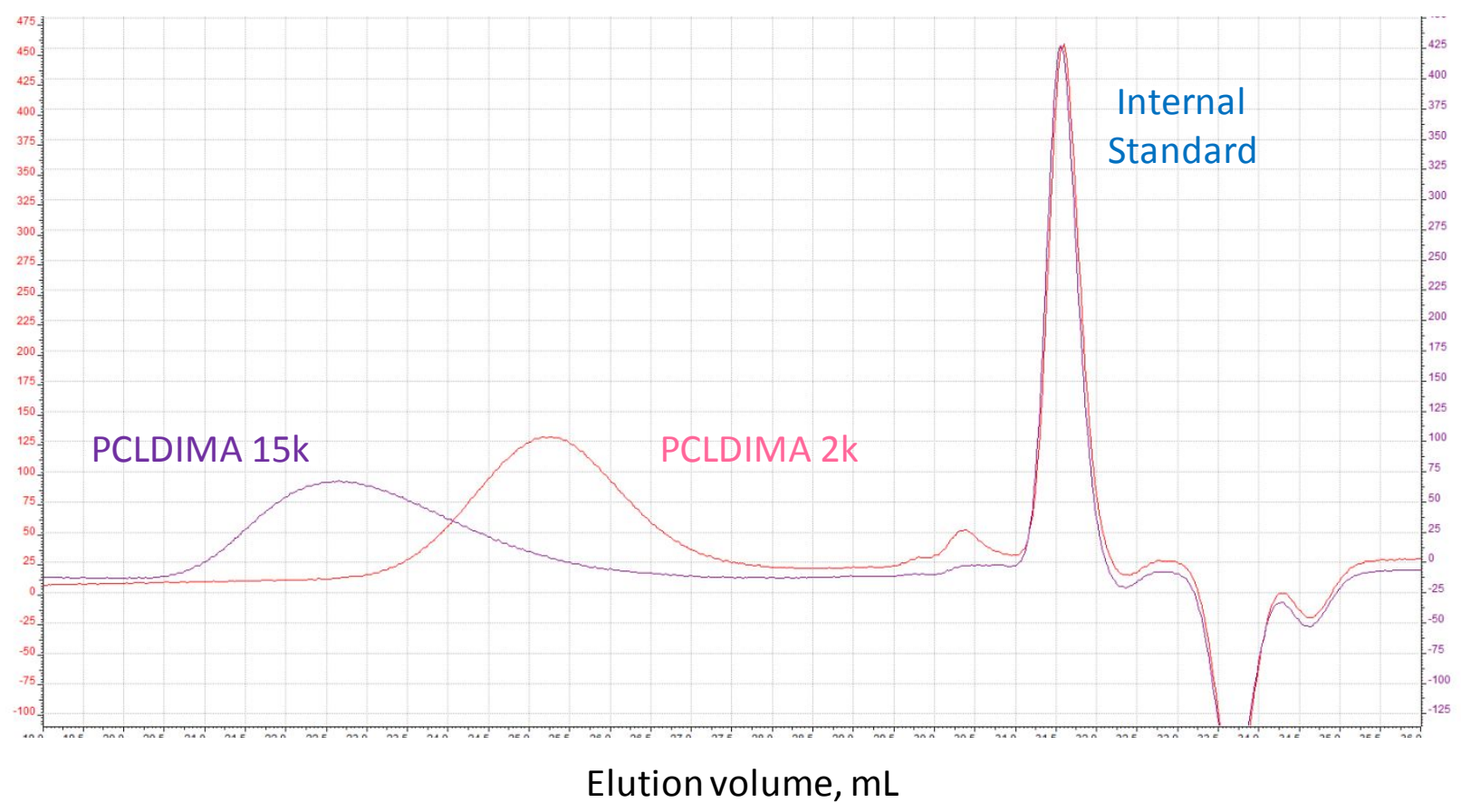

Figure S9. SEC eluograms of PCLDIMA 15k (purple trace) and PCLDIMA 2k (pink trace). THF, $40^{\circ} \mathrm{C}, 0.8 \mathrm{~mL} / \mathrm{min}$. 


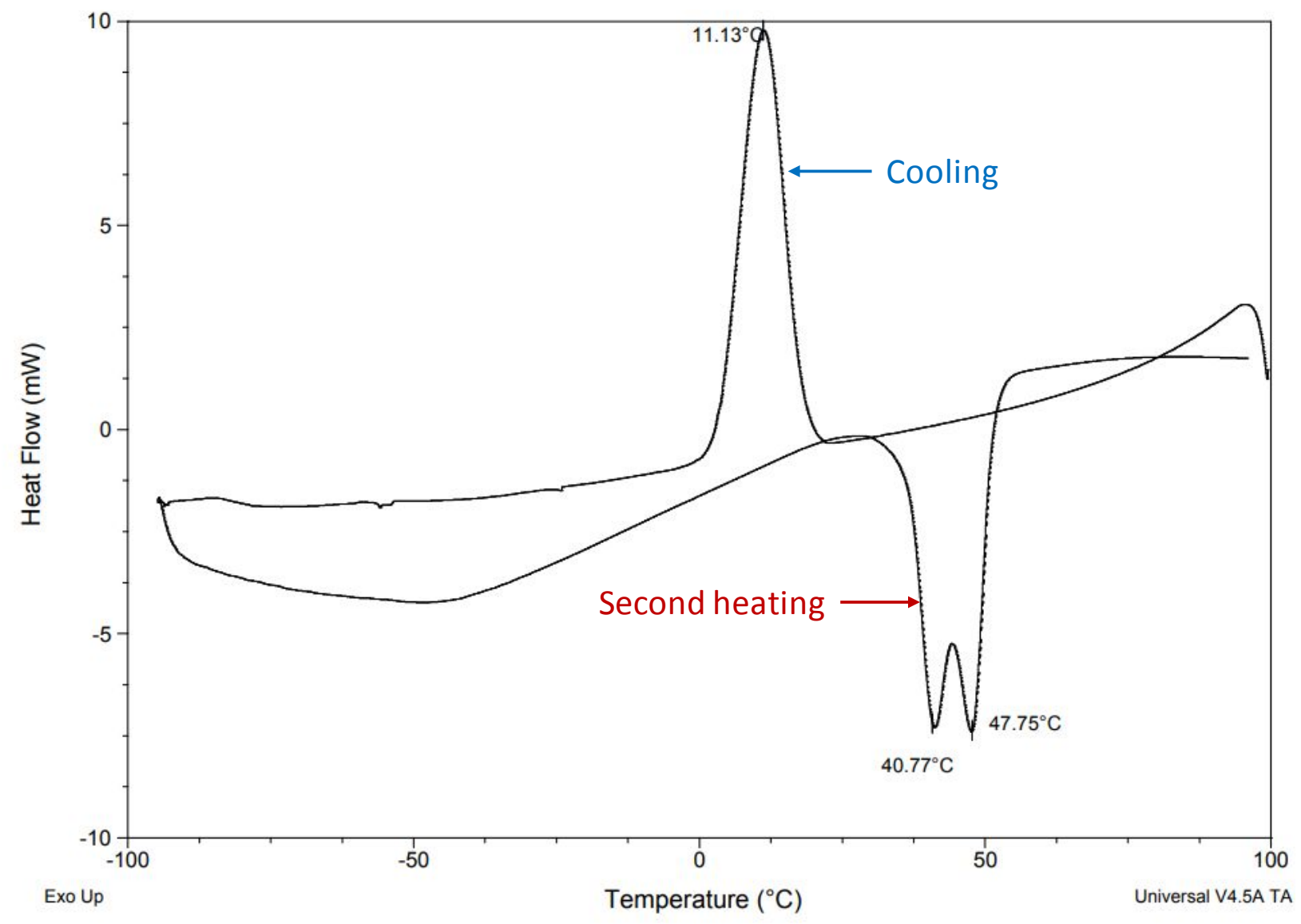

Figure S10. Thermal DSC analysis of PCLDIMA 2k. Heating/cooling rate: $10^{\circ} \mathrm{C} / \mathrm{min}$. 


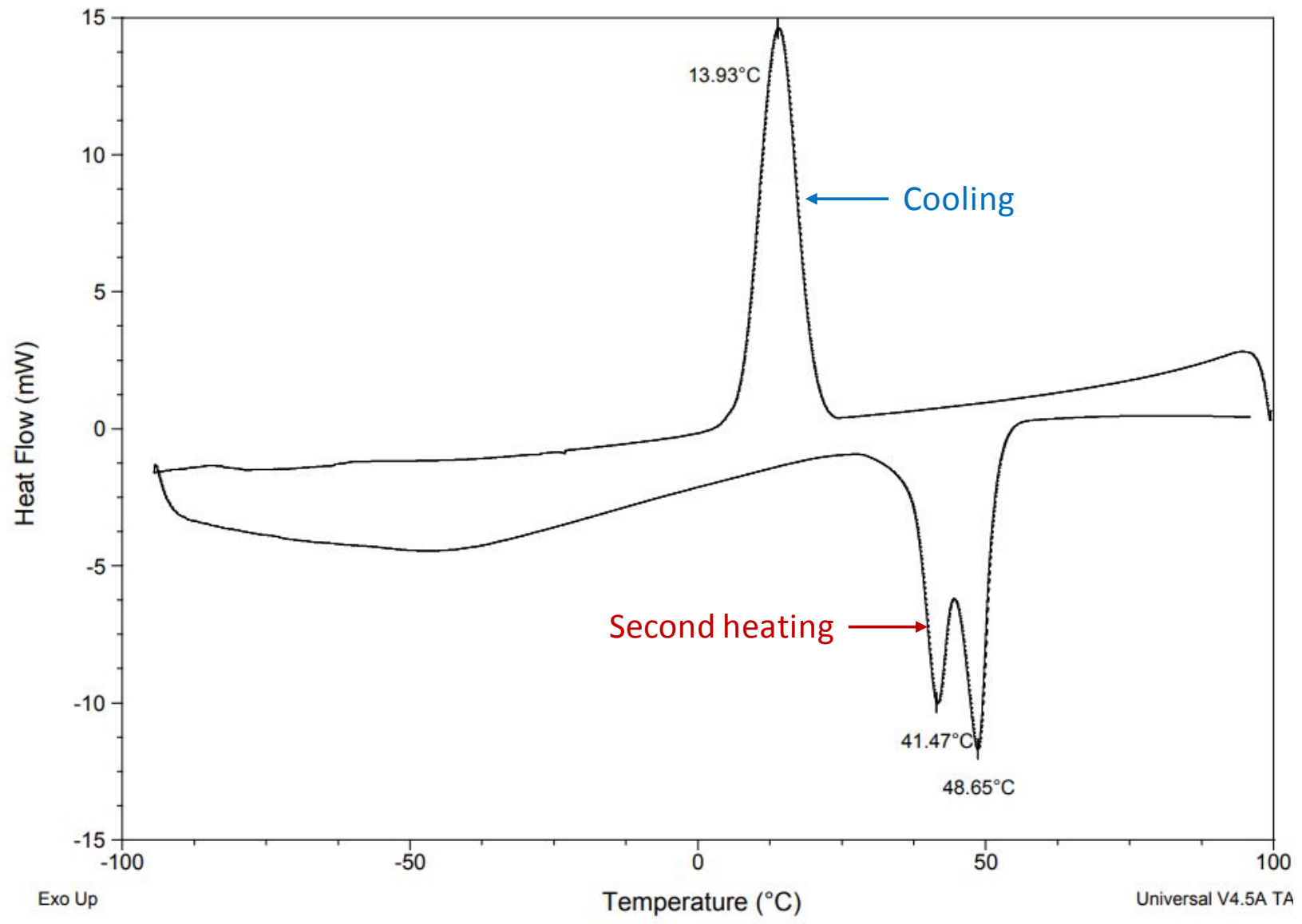

Figure S11. Thermal DSC analysis of PCLDIMA 15k. Heating/cooling rate: $10^{\circ} \mathrm{C} / \mathrm{min}$. 


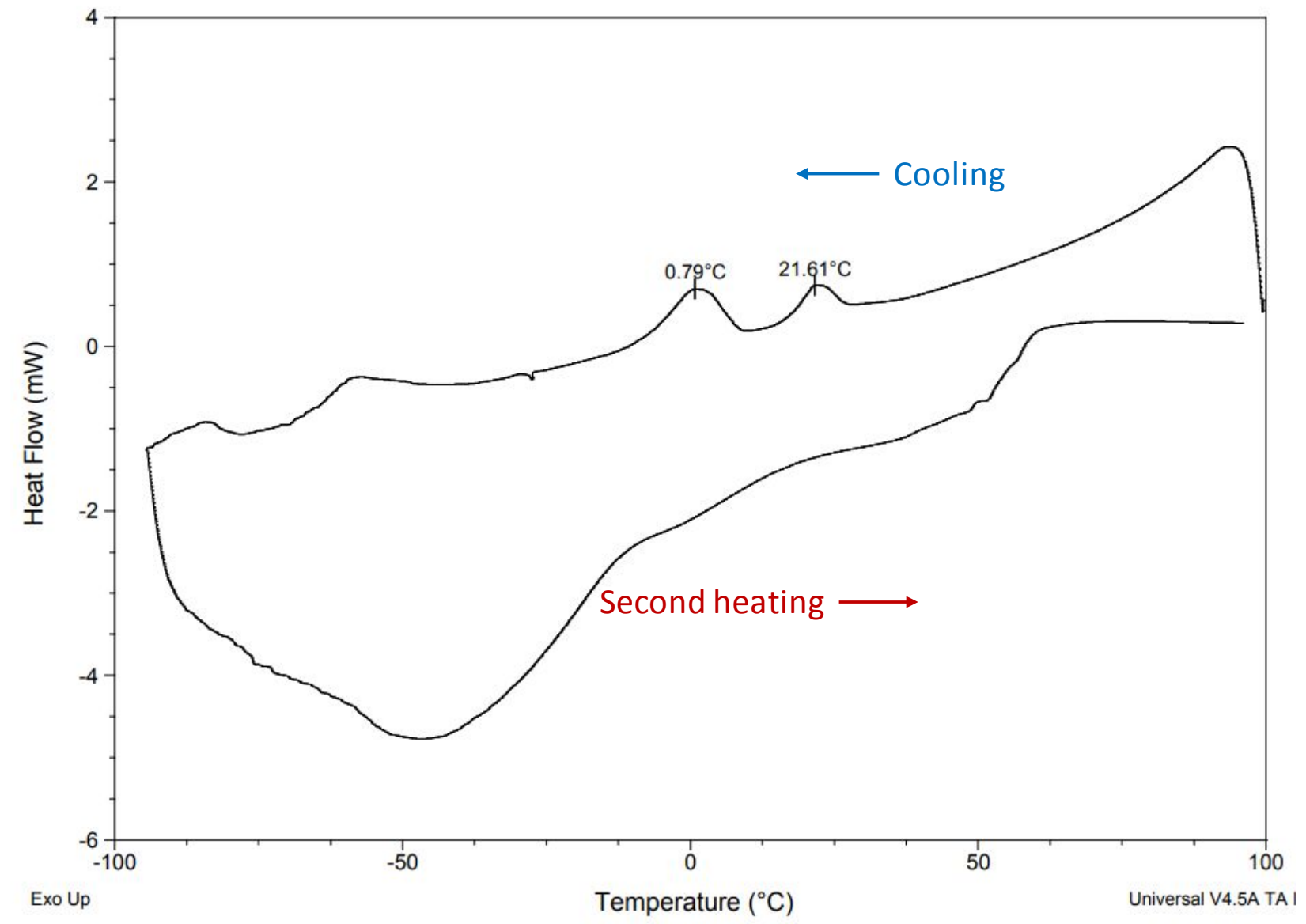

Figure S12. Thermal DSC analysis of rSMP. Heating/cooling rate: $10^{\circ} \mathrm{C} / \mathrm{min}$. 